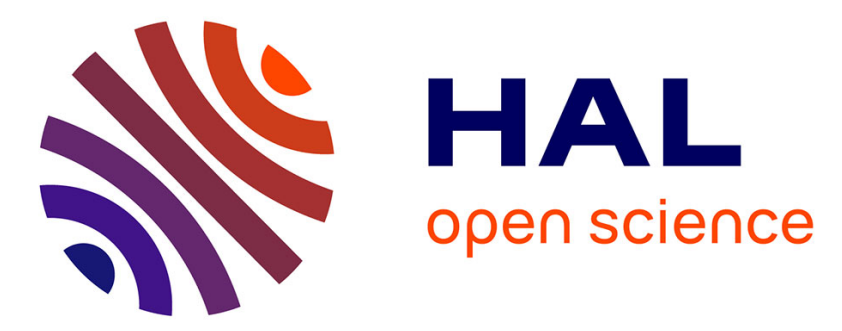

\title{
The optical response above the band gap of light emitting porous silicon, as investigated by photoacoustic spectroscopy
}

\author{
G. Amato, G. Spagnolo, L. Boarino, R. Gavioso, G. Benedetto
}

\section{To cite this version:}

G. Amato, G. Spagnolo, L. Boarino, R. Gavioso, G. Benedetto. The optical response above the band gap of light emitting porous silicon, as investigated by photoacoustic spectroscopy. Journal de Physique IV Proceedings, 1994, 04 (C7), pp.C7-125-C7-128. 10.1051/jp4:1994730 . jpa-00253258

\section{HAL Id: jpa-00253258 https://hal.science/jpa-00253258}

Submitted on 1 Jan 1994

HAL is a multi-disciplinary open access archive for the deposit and dissemination of scientific research documents, whether they are published or not. The documents may come from teaching and research institutions in France or abroad, or from public or private research centers.
L'archive ouverte pluridisciplinaire HAL, est destinée au dépôt et à la diffusion de documents scientifiques de niveau recherche, publiés ou non, émanant des établissements d'enseignement et de recherche français ou étrangers, des laboratoires publics ou privés. 


\title{
The optical response above the band gap of light emitting porous silicon, as investigated by photoacoustic spectroscopy
}

\author{
G. Amato, G. Spagnolo, L. Boarino, R. Gavioso and G. Benedetto \\ Istituto Elettrotecnico Nazionale Galileo Ferraris, Strada delle Cacce 9I, 10124 Turin, Italy
}

\begin{abstract}
Photoacoustic Spectroscopy (PAS) has been performed on Porous Silicon Layers (PSL) obtained by chemical and electrochemical etching of crystalline Silicon. In the investigated energy range $(2.0 \mathrm{eV}-4.7 \mathrm{eV})$ the samples behave as optically opaque and show strong light scattering properties so to prevent the application of standard reflectivity/trasmission techniques. PAS proves suitable in studying porous media, providing evidence that PSLs retain the original cristallinity. The presence of native oxides on PSLs has been revealed by PAS.
\end{abstract}

\section{INTRODUCTION}

Recent observations [1,2] of room temperature visible photoluminescence (PL) from crystalline silicon wafers after anodic oxidation in a hydrofluoric acid based electrolyte in order to obtain the so-called Porous Silicon (PS), have renewed the hopes of achieving silicon integrated optoelectronics.

On the other hand, PSLs can be conveniently used for a variety of applications, namely, antireflective coatings on Si light detectors [3], host for laser dyes [4], passivating layers [5], and gas sensors [6].

PS is often characterized by an array of silicon columns (about 2-50 $\mathrm{nm}$ in diameter) attached to the underlying crystalline silicon substrate, and a large surface to volume ratio. The most accepted explanatory models consider these silicon nanostructures as "quantum wires" with a nearly direct gap, and high probability for radiative carriers recombination. The situation is very different in bulk, crystalline silicon, with indirect gap and a negligible probability for radiative recombination.

Indirect evidence of the strong light scattering properties of PSLs is provided by performing Photothermal Deflection Spectroscopy on a Si wafer, covered by a mesoporous PSL. As Fig. 1 shows, the optical properties of the underlying substrate are fully masked by those of the PSL. The light scattering properties of PS dramatically enhance the light path length so to render the underlying c-Si wafer as opaque even in the infrared. On the contrary, unindefined peaks, probably due to IR vibrational overtones can be detected. A possible way to undergo optical characterization on PSLs is to obtain free-standing films [2]. This procedure, however requires difficult sample preparation steps.

In order to perform a detailed characterization of the optical and structural properties of PSLs attached to the original substrate, PAS has been employed in the visible-UV range.

PAS is a very well established technique to study the optical and thermal properties of semiconducting materials. In the limit of fully opaque samples $\left(\alpha^{-1}<t\right.$, where $\alpha$ is the optical absorption coefficient and $t$ is the sample thickness) the PAS signal depends only on the sample surface reflectivity $R$, being proportional to 1-R. PAS is easily applicable to PSLs, since it detects the amount of absorbed light, which is high in the case of porous material, instead of the small percentage of reflected light.

\section{EXPERIMENTAL}

PSLs have been produced by anodization of non-degenerate p-type c-Si wafers $(\rho=10 \Omega \mathrm{cm})$ and by simple stain etching of degenerate p-type wafers $(\rho=0.01 \Omega \mathrm{cm})$. In both cases, a HF-based electrolyte 
has been used. Details on the preparation methods can be found elsewhere [2,7]. The material porosity has been measured by means of the gravimetric method [7] assuming the weight change to be due solely to loss of Si. All these steps have been performed in a clean room, to minimize contamination of the PSLs.

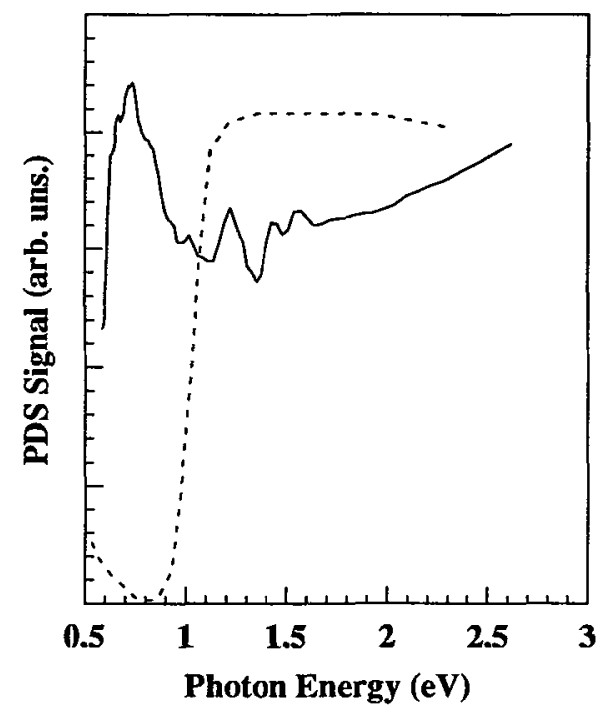

Fig. 1. The PDS spectrum of a Si wafer with a $1.5 \mu \mathrm{m}$ thick PSL (full curve). The spectrum of a clean wafer is shown for comparison (dashes)

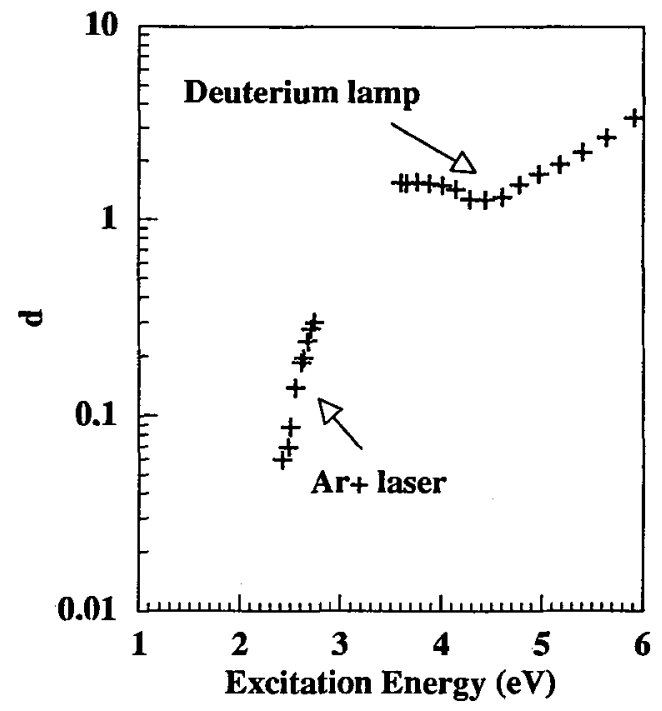

Fig. 2. PLE spectrum of a typical PSL. The excitation sources are indicated.

PAS measurements have been carried out in a standard apparatus, equipped with a Xe lamp, at a modulation frequency of $15 \mathrm{~Hz}$, in the energy range from $2.0 \mathrm{eV}$ to $4.7 \mathrm{eV}$. An optimized PAS cell, with a $\mathrm{CaF}_{2}$ optical window has been used

Optical gap measurements on free-standing films have been performed [2], reporting values of the order of $1.8 \mathrm{eV}$ and 1.2 for high and low porosity material, respectively. This agrees with the quantum wire model which assumes that the optical gap of the wires is inversely related to their diameter $L$.

In order to gain more insight on the optical absorption properties of PSLs, Photoluminescence Excitation Spectroscopy (PLE) has been carried out. The PLE signal can be related to the optical absorption by means of:

$$
\mathrm{IPL}_{\mathrm{P}} \approx\left[\mathrm{I}_{0}\left(1-\mathrm{e}^{-\alpha \mathrm{d}}\right)\right]^{\mathrm{n}}
$$

where IPL is the integrated PL intensity, $I_{0}$ is the incident intensity, $d$ is the film thickness, and $n$ is a factor related to the recombination mechanism. It has to be noted that $d$ is the optical thickness of the film and is $\mathrm{d} \gg>t$, because of the scattering phenomena. A sharp excitation edge at $h v>2 \mathrm{eV}$, is reported in fig. 2. The PLE excitation edge, corresponds, in the framework of the quantum wire model, to the optical gap of luminescing nanostructures, say, to those having smaller diameter. It is thus expected that the averaged optical gap is smaller than the excitation edge. As a consequence, we can assume that in the investigated range, most of the nanostructures efficiently absorb the incident radiation.

From simple reasonning related to effective mass approximation and light scattering theory we can calculate the light penetration depth in mesoporous $(L \sim \lambda)$ and microporous $(L<<\lambda)$ samples to be below $1 \mu \mathrm{m}$ and $5 \mu \mathrm{m}$, respectively, in all the investigated range. To ensure a negligible contribution from the Si substrate, mesoporous, $\leq 60 \%$ porosity, stain etched, $1.7 \mu \mathrm{m}$ thick PSLs and microporous, $\sim 80 \%$ porosity, anodically etched, $7 \mu \mathrm{m}$ thick PSLs, have been investigated.

All the PAS measurements have been performed in ambient air. PSLs are suspected to rapidly oxidize when kept in air, as a consequence for the high surface-to-volume ratio. For this reason, and to guarantee stability under measurements, the samples where all aged for more than 1 month before investigation. 


\section{RESULTS}

Fig. 3 reports some of the results as obtained on PSLs of $30 \%$ and $60 \%$ porosity. For comparison, the PAS spectrum as obtained on polished, clean Si wafer, is shown. All the PAS spectra have been normalized to the reflectivity value, as measured at $633 \mathrm{~nm}$ by means of a He-Ne laser and an integrating sphere.
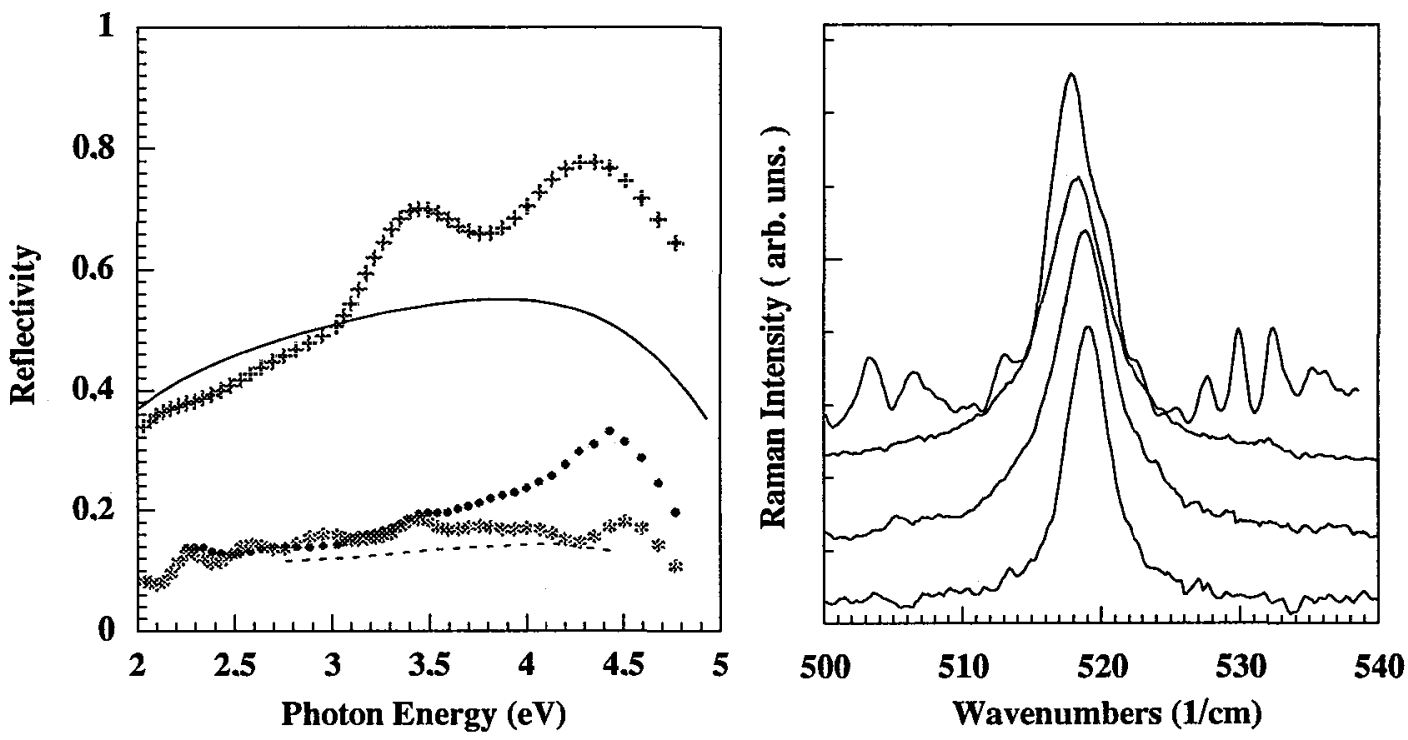

Fig.3. Reflectivity curves, as obtained by PAS, of PSLs with porosity $30 \%(\bullet)$ and $60 \%(*)$ and $0(c-$ $\mathrm{Si},+)$. The reflectivity of a-Si (full curve) and $\mathrm{SiO}$ Fig. 4. Raman spectra of samples of various porosity. From top to bottom: $80 \%, 60 \%, 30 \%$ and 0 (c-Si).

(dashes), as calculated from the data of ref. 6 is also reported.

The structures at $\sim 3.4 \mathrm{eV}$ and $\sim 4.5 \mathrm{eV}$, are evident in the case of crystalline silicon. Fig. 3 shows that the PS layers mantain these peaks at these energies, meaning that the crystalline character of the starting material is almost conserved. This is confirmed by the Raman spectra in Fig. 4 which show a small broadening of the spectra when porosity is increased to $60 \%$.

An amorphous phase can be assumed to be present in PS samples. As a matter of fact, when increasing porosity the contrast of the peaks is reduced, meaning a relaxation of the crystalline network. a-Si, if present, should have a broad Raman emission at $\sim 480 \mathrm{~cm}^{-1}$. This emission has not been observed in our experiment, thus it could be concluded that the amorphous-like behaviour of $R$ in the case of samples with $60 \%$ porosity is attributable to an oxide phase. Effective medium approximation is currently carried out to determine the amount of these different phases in PS, and to correlate them with the porosity degree.

The above analysis for PAS spectra could not however be applied in the case of a $80 \%$ porosity, electrochemically etched PS sample. Figs. 5 and 6 compare the PAS signal and phase vs. energy for two typical PSLs, $60 \%$ and $80 \%$ porosity, respectively. It is found that when porosity is increased to a value of the order of $60 \%$, the PAS response is dictated solely by the surface reflectivity. The sample behaves in this case like a bulk material, and no appreciable phase shift is detected. On the contrary, a phase shift of $\sim 25^{\circ}$ is observed in the case of $80 \%$ porosity sample, indicating that different sample portions are investigated when decreasing the light penetration depth. Correspondingly to the phase shift, a noticeable variation of the PAS signal is detected. Variations in the PS optical parameters can hardly explain a drastic reflectivity decrease at $\sim 3.5 \mathrm{eV}$.

The same effect can be obtained when investigating an ad hoc sample, as obtained by growing $0.3 \mu \mathrm{m}$ thermal oxide on c-Si. Thermal oxide is well known to reduce the sample reflectivity in the full visible-UV range. Nevetheless, PAS analysis, (figs. 5 and 6) shows a steep decrease of the signal at energies typical of the $\mathrm{SiO}_{2}$ optical gap, associated to a phase shift of $\sim 45^{\circ}$. This is typical of a transition from a surface absorption to a bulk absorption regime. At high energies, $\mathrm{SiO}_{2}$ absorbs and the photothermal signal is generated on the surface. When $\mathrm{SiO}_{2}$ becomes transparent, the optical absorption occurs in the substrate. 
The thermal conductivity mismatch at the $\mathrm{Si}_{-} \mathrm{SiO}_{2}$ interface excellently explains both the phase lag and the drastic reduction of the signal amplitude.

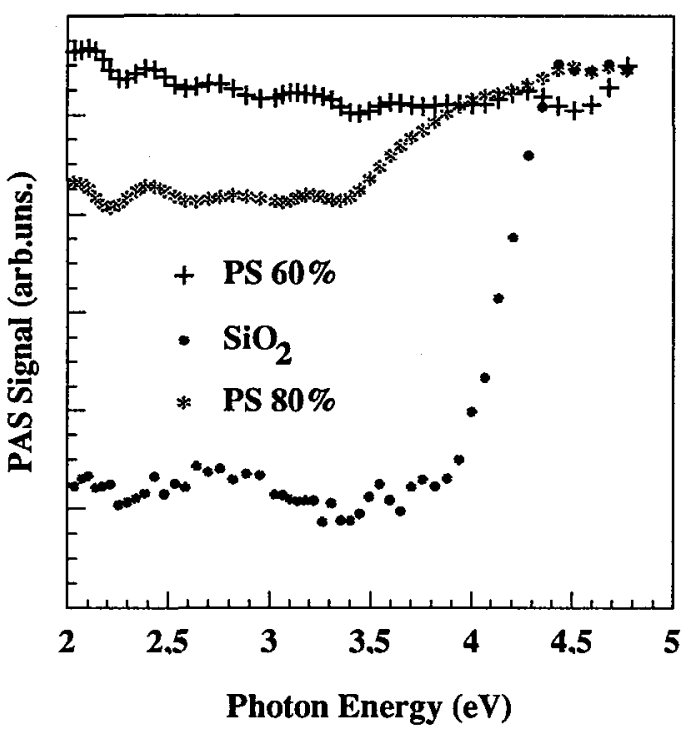

Fig. 5. The PAS signal of two typical PS samples on $\mathrm{c}-\mathrm{Si}$, compared with that of a c-Si wafer, with a $2 \mu \mathrm{m}$ thermal oxide layer.

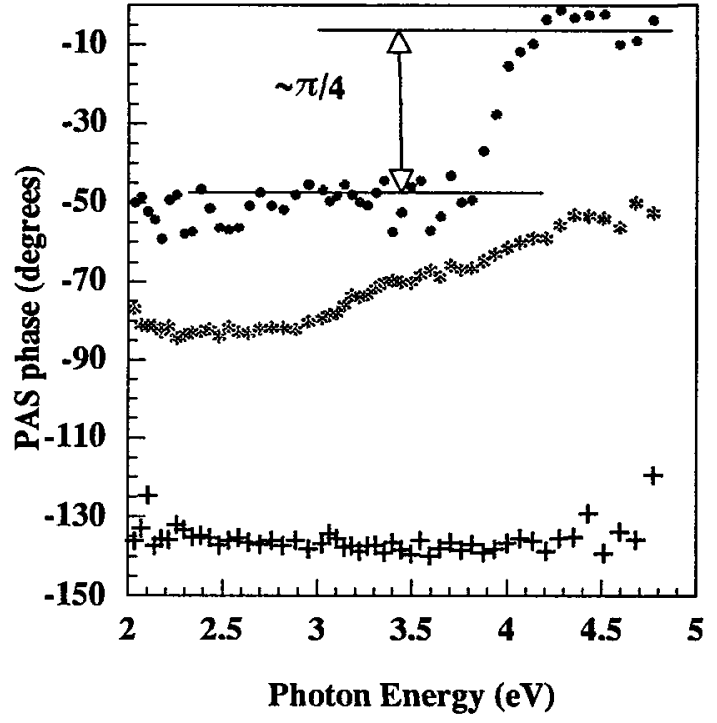

Fig. 6. The PAS phase for the cases of the previous fig.

We suggest that a similar mechanism occurs also in the high porosity PSLs. IR vibrational spectroscopy data indicate strong oxidation of high porosity PSLs when kept in air for some weeks. In the case of mesoporous, stain etched PS, however, the oxide phase affects solely the sample reflectivity, suggesting a mixture with the c-Si and void phases. In anodically etched, microporous material, PAS data suggest a layered structure, with the oxide on the top of the film. This is consistent with the observation that smaller wires (L 2-5 nm), which at the top of the PSL, can be fully oxidized in a more easy way. It has to be underlined, on the other hand, that the different preparation methods and the different doping of the starting wafers can play a role in affecting the PS composition and stability.

\section{CONCLUSIONS}

This paper reports on preliminary applications of PAS in the vis.-UV range to Porous Silicon layers. The results indicate that the PSLs retain the original wafer crystallinity, and show that the oxidation level increases when increasing porosity. PAS reveals unsuspected capabilites in discriminating between different phases in high porosity samples, allowing for depth profiling analyses. These can be of great importance in the study of this new, fascinating material.

Acknowledgements. The authors are indebted with V. Bullara for the technical assistance.

\section{References}

[1] L. T. Canham, Appl. Phys. Lett. 57, (1990) 1046.

[2] V. Lehmann and U. Gosele, Appl. Phys. Lett. 58, (1991), 856.

[3] J. P. Zheng, K.L. Jiao, W.P. Shen, W.A. Anderson, H.S. Kwok, Appl. Phys. Lett. 61 (1992), 459

[4] L. T. Canham, Appl. Phys. Lett. 63, (1993) 337.

[5] C. Pickering, M.I.J. Beale, D.J. Robbins, P.J. Parson, R. Greef, J. Phys. C, 17 (1984), 6535.

[6] J. M. Lauerhaas, G. M. Credo, J.L. Heinrich and M. Sailor, J. Am. Chem. Soc., 114 (1992), 1911.

[7] G. Amato, submitted for publication on Jap. Jour. Appl. Phys. 